\title{
Rashba Splitting and Dichroism of Surface States in Bi/Ag Surface Alloy
}

\author{
Guang Bian, T. Miller, and T.-C. Chiang* \\ Department of Physics, University of Illinois at Urbana-Champaign, 1110 West Green Street, \\ Urbana, Illinois 61801-3080, USA \\ Frederick Seitz Materials Research Laboratory, University of Illinois at Urbana-Champaign, \\ 104 South Goodwin Avenue, Urbana, Illinois 61801-2902, USA
}

\begin{abstract}
The Rashba effect plays an important role in various spin-related phenomena in two-dimensional electronic systems. In this work we present a theoretical analysis of the Rashba effect both analytically and numerically for the prototypical Rashba system Bi/Ag surface alloy, which shows a giant Rashba spin splitting. The results reveal the critical influence of atomic spin-orbit coupling and structural inversion asymmetry. In addition, we demonstrate a theoretical route to interpret the prominent circular dichroic patterns observed by angle-resolved photoemission spectroscopy in this system. The results reveal a close connection between the experimentally observed dichroic patterns and the Rashba spin texture.
\end{abstract}

Keywords: Rashba effect, Bi/Ag surface alloy, ARPES, circular dichroism

* Email address: tcchiang@illinois.edu (T.-C. Chiang) 


\section{Introduction}

The Rashba effect is a combined effect of atomic spin-orbit coupling and structural inversion asymmetry (SIA) in crystal potentials. Generally it causes a momentum-dependent spin splitting of electronic bands in condensed matter systems [1]. It is ubiquitous in various two-dimensional systems such as surfaces and heterostructures. This spin splitting effect is usually weak since it is derived from a relativistic correction to the spinless Hamiltonian of band theory. However, significant or large Rashba splittings have been observed in some cases such as the surface states of $\mathrm{Au}(111)$ and $\mathrm{Bi} / \mathrm{Ag}$ surface alloys $[2,3,4,5,6,7,8]$. The same interaction is responsible for the spin polarization of the boundary states in $2 \mathrm{D}$ and $3 \mathrm{D}$ topological systems $[9,10]$. All of these systems share two common features, a strong atomic spin-orbit coupling (SOC) of heavy atoms (such as $\mathrm{Au}$ and $\mathrm{Bi}$ ) and SIA that gives rise to an electric field perpendicular to the surfaces and interfaces.

Systems with large Rashba spin splittings are of interest for the development of spintronics-based devices. In addition to experimental searches for Rashba systems, there have been extensive theoretical efforts to describe the Rashba phenomena. Density functional theory, $\mathbf{k} \cdot \mathbf{p}$ band theory, tight-binding models, and orbital-angular momentum (OAM) analysis have been employed to interpret the spin splitting $[11,12,13,14,15]$. While these numerical or empirical approaches are highly successful, the underlying physics is often obscured by 
numerical complications or the use of adjustable parameters. It is interesting to examine physically the relationship between the Rashba splitting and the two defining factors, the atomic SOC and SIA, at the most basic level. In this work we presented such an analysis for a prototypical Rashba system $\mathrm{Bi} / \mathrm{Ag}$ surface alloy. The strengths of SOC and SIA are varied theoretically to establish the functional relationship that is useful as a guide for designing strong Rashba systems.

It is well recognized that the spin polarization of Rashba surface states can give rise to prominent dichroic patterns as observed by angle resolved photoemission spectroscopy (ARPES) $[16,17,18,19,20]$. However, the direct connection between the dichroic patterns and spin texture has remained a challenge. The difficulties lie in the photoemission process that can involve multiple mechanisms that depend on the photon energy. In the second part of this paper, we present a parameter-free analysis for the dichroic signal of the surface states in the $\mathrm{Bi} / \mathrm{Ag}$ surface alloy [21]. The data were taken under experimental conditions that emphasize surface photoemission over bulk photoemission, which leads to consideration simplification of the analysis. The results demonstrate an unambiguous connection of the spin texture of $\mathrm{Bi} / \mathrm{Ag}$ surface alloy and the observed dichroic patterns.

\section{Theoretical interpretation of Rashba spin splitting in $\mathbf{B i} / \mathbf{A g}$}

One third of a monolayer (ML) of $\mathrm{Bi}$ alloyed into a $\operatorname{Ag}(111)$ surface yields free-electron-like surface state bands with the largest known Rashba spin splitting [3,4]. Figures 1(a)-(c) show the sample configuration used in the experiment and the lattice structure of the 
Bi/Ag surface alloy. The $1 / 3 \mathrm{ML}$ of Bi organizes into a $\sqrt{3} \times \sqrt{3}$ structure in the top atomic layer. The $\mathrm{Bi}$ atoms are located higher than the $\mathrm{Ag}$ atoms in the top atomic plane by $\Delta z=0.65 \AA$ [7]. This is defined as the buckling parameter in our analysis below, and it is a measure of the local SIA. The effects of Bi buckling on the spin splitting can be intuitively understood as follows. Without buckling, the spin splitting should be zero because of structural inversion symmetry of a flat $\mathrm{Bi} / \mathrm{Ag}$ atomic layer (the $\mathrm{Ag}$ substrate has negligible effect on the spin splitting and thus can be ignore here for simplicity [15]). A structural buckling breaks the inversion symmetry and gives rise to a spin splitting. The calculated surface energy as a function of the buckling parameter shows a minimum at $\Delta z_{\text {cal }}=0.61 \AA$ which is consistent with the experimental observation [7,15]. A typical angle-resolved photoemission spectrum taken using $22 \mathrm{eV}$ photons is presented in Fig. 1(d) [15]. It shows at below the Fermi level a pair of hole-like Rashba-split surface bands centered about $\bar{\Gamma}$. Additional faint features correspond to another set of Rashba-split surface bands with their crossing point (Dirac point) above the Fermi level; the portions of the bands above the Fermi level, not observed by photoemission, are indicated by dashed curves as a guide to the eye based on a comparison with theoretical DFT calculations presented in Fig. 2(a). The lower set of surface states has mainly $s p_{z}$ character at the Bi site, while the upper set has almost pure Bi $p_{x, y}$ character. The energy dispersion relations for these bands near the $\Gamma$ point can be described by

$$
E\left(\boldsymbol{k}_{\|}\right)=\frac{\hbar^{2}}{2 m^{*}}\left(k_{\|} \pm \Delta k\right)^{2}+E_{0}
$$

where $m^{*}$ is the effective mass, $\Delta k$ is the offset of the free-electron-like bands for the two 
opposite spin directions, and $E_{0}$ is a constant. The mutually opposite spin directions of the two bands are perpendicular to both $\mathbf{k}_{\|}$and $\hat{\mathbf{e}}_{z}$. The computed spin splitting $\Delta k$ of the $p_{x, y}$ bands as a function of the buckling parameter $\Delta z$ is shown in Fig. 2(b). It has a maximum at about the experimentally observed buckling.

(a)

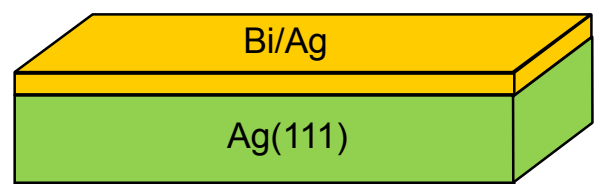

(b)

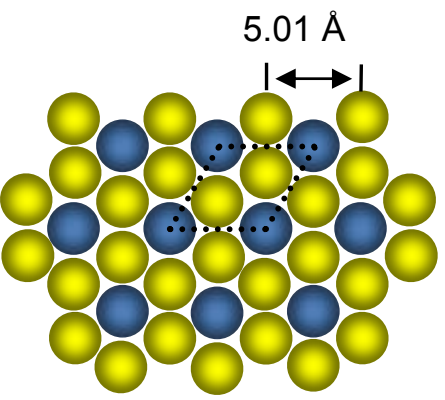

(c)

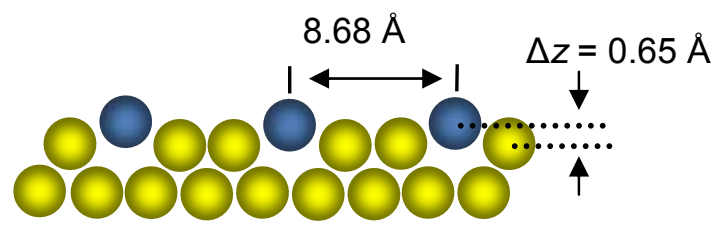

(d)

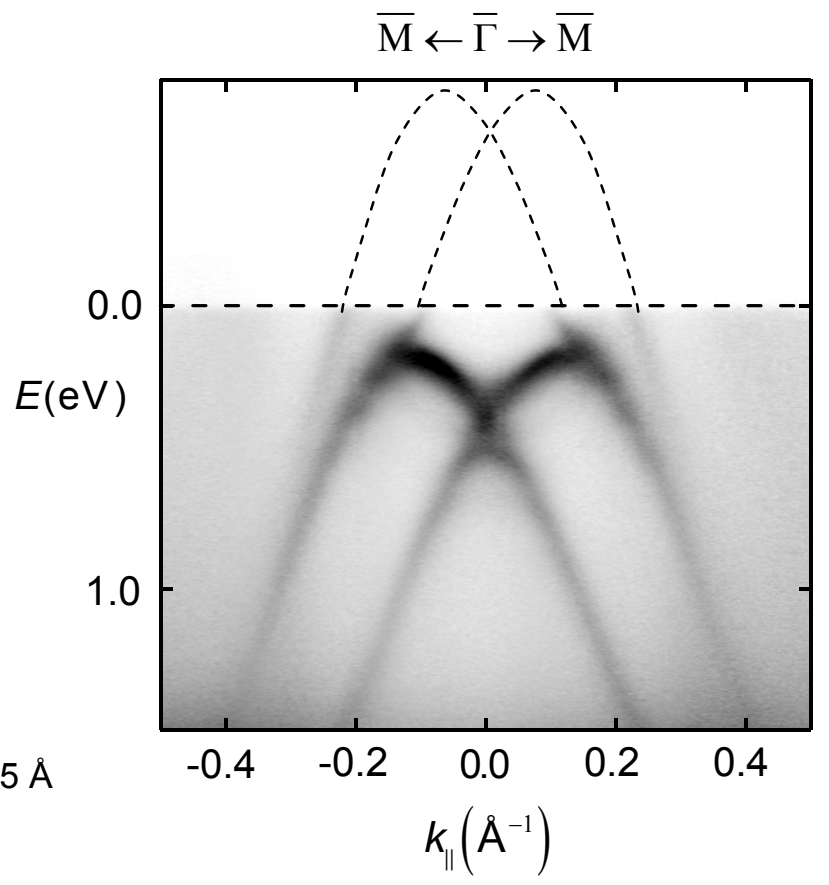

FIG. 1. (a) Schematic structure of the Bi/Ag surface alloy used in the experiment. (b) Top view of the atomic structure. (c) Side view. Blue balls indicate Bi atoms and yellow balls stand for Ag atoms. (d) A photoemission spectrum from a Bi/Ag surface alloy along the $\bar{\Gamma}-\overline{\mathrm{M}}$ direction taken with $22-\mathrm{eV}$ photons. The dashed curves are a guide to the eye for the upper Rashba split surface bands. 


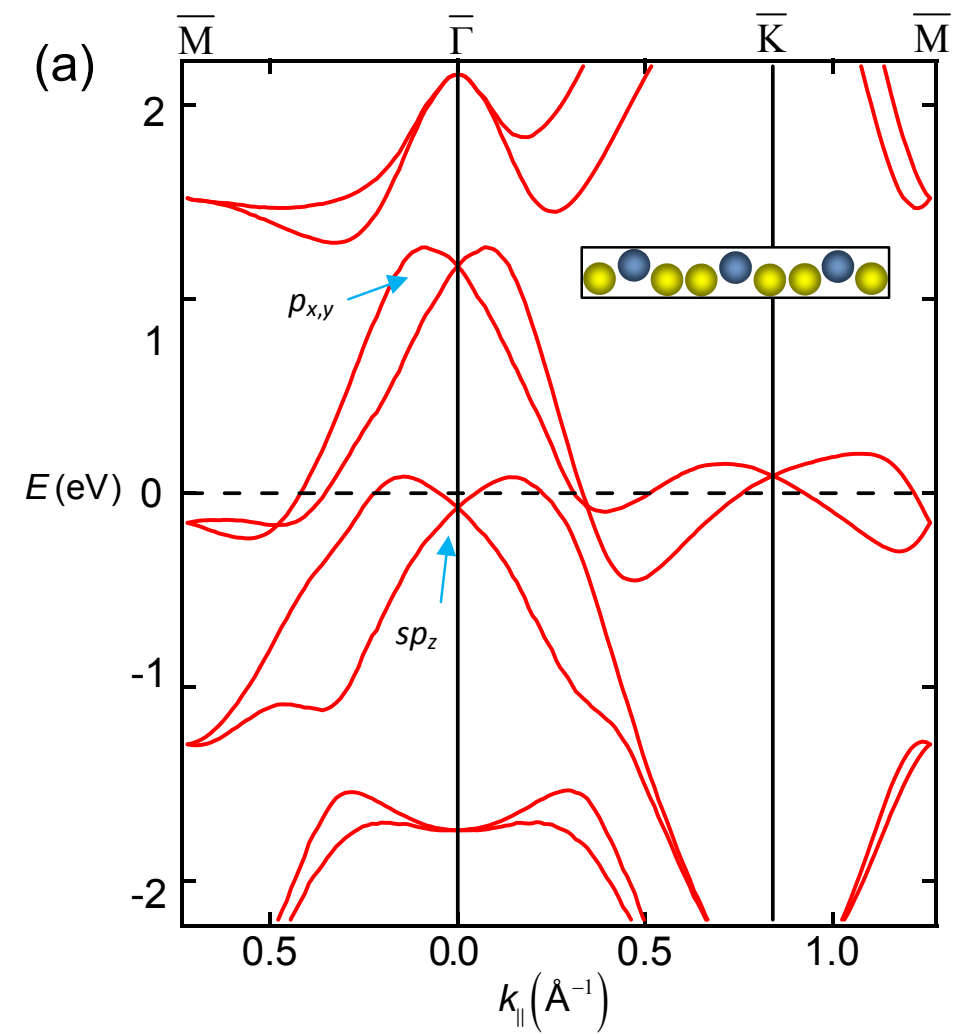

(b)

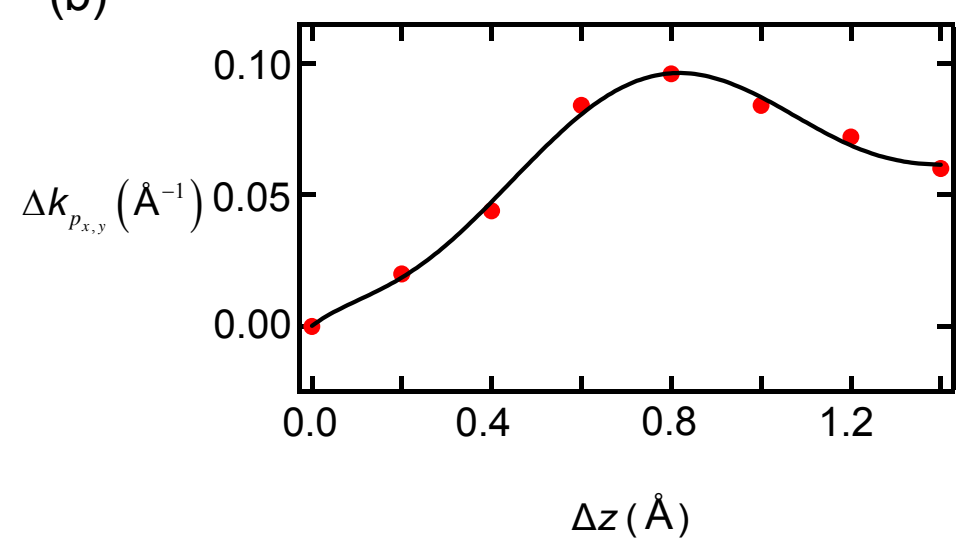

FIG. 2. (a) Calculated band structure of an isolated buckled layer of $\mathrm{Bi} / \mathrm{Ag}$. (b) Calculated Rashba momentum shift in the $p_{x, y}$ bands as a function of $\Delta z$.

Prior work has shown that the surface states in $\mathrm{Bi} / \mathrm{Ag}$ are well localized within the top monolayer where $\mathrm{Bi}$ and $\mathrm{Ag}$ form an alloy [15]. The band dispersion relations and the spin 
splittings of these surface states are largely unchanged with or without the Ag substrate, and the Rashba effect for the pure $\operatorname{Ag}(111)$ surface itself is negligibly small. For simplicity, we will present in the following an analysis for the monolayer alloy system. Generalization to include a substrate is straightforward.

The SOC for an electron in a potential energy field $V$ is given by

$$
H_{L S}=\frac{\hbar}{4 m^{2} c^{2}} \boldsymbol{\sigma} \cdot \nabla V \times \mathbf{p}=\frac{e \hbar}{4 m^{2} c^{2}} \boldsymbol{\sigma} \cdot \mathbf{E} \times \mathbf{p}
$$

where $e$ is the magnitude of the electronic charge (positive), and $\mathbf{E}=\nabla V / e$ is the electric field associated with $V$. In solids or atoms, the interaction is dominated by the strong Coulomb field in the inner core region around each atomic nucleus, and so the electric field can be taken to be radial.

$$
\mathbf{E}=\frac{1}{e} \nabla V=\frac{1}{e} \frac{d V(r)}{d r} \frac{\mathbf{r}}{r}
$$

The interaction can be rewritten as

$$
H_{L S}=\frac{1}{2 m^{2} c^{2}} \frac{1}{r} \frac{d V(r)}{d r} \mathbf{s} \cdot \mathbf{l}=\frac{1}{2 m^{2} c^{2}} \frac{1}{r} \frac{d V(r)}{d r} \mathbf{s} \cdot \mathbf{r} \times \mathbf{p}
$$

where $\mathbf{I}$ and $\mathbf{s}$ are the orbital and spin angular momenta, respectively. This interaction is stronger for heavier atoms because of the stronger nuclear Coulomb field (it can be shown that the atomic spin-orbit splitting is roughly proportional to $Z^{4}$ ). For the $\mathrm{Bi} / \mathrm{Ag}$ alloy, we need to consider the interaction associated with the Bi atoms only. The total one-electron Hamiltonian is given by 


$$
H=\frac{p^{2}}{2 m}+V(\mathbf{r})+\frac{e \hbar}{4 m^{2} c^{2}} \boldsymbol{\sigma} \cdot \mathbf{E} \times \mathbf{p}=\frac{p^{2}}{2 m}+V(\mathbf{r})+\frac{\hbar}{4 m^{2} c^{2}} \boldsymbol{\sigma} \cdot \nabla V \times \mathbf{p}
$$

Following the $\mathbf{k} \cdot \mathbf{p}$ method for Bloch states in a periodic lattice, the Hamiltonian for the periodic part of the Bloch wave function is

$$
\begin{gathered}
H_{\mathbf{k}}=\frac{p^{2}}{2 m}+\frac{\hbar \mathbf{k} \cdot \mathbf{p}}{m}+\frac{\hbar^{2} k^{2}}{2 m}+V(\mathbf{r})+\frac{e \hbar}{4 m^{2} c^{2}} \boldsymbol{\sigma} \cdot \mathbf{E} \times(\mathbf{p}+\hbar \mathbf{k}) \\
H_{\mathbf{k}}=H_{0}+H_{\mathbf{k}}^{\prime}=\left(\frac{p^{2}}{2 m}+V(\mathbf{r})+\frac{e \hbar}{4 m^{2} c^{2}} \boldsymbol{\sigma} \cdot \mathbf{E} \times \mathbf{p}\right)+\left(\frac{\hbar \mathbf{k} \cdot \mathbf{p}}{m}+\frac{\hbar^{2} k^{2}}{2 m}+\frac{e \hbar}{4 m^{2} c^{2}} \boldsymbol{\sigma} \cdot \mathbf{E} \times \hbar \mathbf{k}\right)
\end{gathered}
$$

where $H_{0}$ is the Hamiltonian at the zone center. $H_{\mathbf{k}}{ }^{\prime}$ is treated as a perturbation here. Its last term only is spin-dependent. It has the familiar (phenomenological) Rashba form, but it is dominated by the strong electric field in the inner core regions. Electric fields in the interstitial region are generally negligible for this purpose.

Consider an atomic layer of $\mathrm{Bi}-\mathrm{Ag}$ with variable buckling. The valence states at the zone center are spin degenerate, with or without buckling. These are eigenstates of $H_{0}$, and are taken as the unperturbed states:

$$
\left|\psi_{0, \pm}\right\rangle=\left|u_{0}(\mathbf{r}) \chi_{ \pm}\right\rangle
$$

where $\chi$ is the spin part of the wave function with the quantization axis chosen along $y$, and $u_{0}$ is a periodic function. The spin splitting is identically zero for an unbuckled layer because of the inversion symmetry of the system. The degeneracy at finite $k_{x}$ is lifted to first order with a finite buckling: 


$$
\Delta \mathcal{E}\left(k_{x}\right)=\left\langle\psi_{0,+}\left|H_{\mathbf{k}}^{\prime}\right| \psi_{0,+}\right\rangle-\left\langle\psi_{0,-}\left|H_{\mathbf{k}}^{\prime}\right| \psi_{0,-}\right\rangle=2\left\langle u_{0}\left|\frac{e \hbar}{4 m^{2} c^{2}} \hat{\mathbf{y}} \cdot \mathbf{E} \times \hbar \mathbf{k}\right| u_{0}\right\rangle=\frac{e \hbar^{2} k_{x}}{2 m^{2} c^{2}}\left\langle u_{0}\left|E_{z}\right| u_{0}\right\rangle(9)
$$

The splitting is proportional to $k_{x}$ to first order (leading to a Dirac cone). The integral is dominated by the region near the nucleus. There, the electric field $E_{z}$ for an unbuckled layer is given by

$$
E_{z}(\mathbf{r})=\frac{-Z e}{r^{3}} z
$$

This is an odd function and the integral is zero (no splitting). With buckling, the center of symmetry of $E_{z}$ moves upward relative the unperturbed wave function.

$$
E_{z}(\mathbf{r}-\hat{\mathbf{z}} \Delta z)=E_{z}(\mathbf{r})-\Delta z \frac{\partial E_{z}}{\partial z}=E_{z}(\mathbf{r})-\Delta z\left[\frac{-Z e}{r^{3}}+3 \frac{Z e}{r^{4}} z^{2}\right]
$$

The first term corresponds to the nonbuckled case and does not lead to a splitting. For the inner core region, the third term, of the order of $r^{-2}$, can be ignored relative to the second term, which is proportional to $r^{-3}$.

$$
\Delta \mathcal{E}\left(k_{x}\right)=\frac{e \hbar^{2} k_{x}}{2 m^{2} c^{2}}\left\langle u_{0}\left|E_{z}\right| u_{0}\right\rangle=\frac{e \hbar^{2} k_{x}}{2 m^{2} c^{2}}\left\langle u_{0}\left|\frac{Z e}{r^{3}}\right| u_{0}\right\rangle \Delta z=\frac{Z e^{2} \hbar^{2} k_{x} \Delta z}{2 m^{2} c^{2}}\left\langle u_{0}\left|\frac{1}{r^{3}}\right| u_{0}\right\rangle
$$

For comparison, consider a single $\mathrm{Bi}$ III ion $\left(\mathrm{Bi}^{2+}\right)$ in free space. Its ground configuration is $6 s^{2} 6 p$, where just one $6 p$ valence electron is present outside a filled core. Instead of a neutral atom, we choose this configuration to avoid the complication of coupling to the other $6 p$ electrons in a neutral atom. The relevant term is ${ }^{2} \mathrm{P}_{0}$. Based on the NIST atomic data base [22], the splitting between $6 p_{3 / 2}$ and $6 p_{1 / 2}$ is $2.6 \mathrm{eV}$ for this single electron. The relevant interaction 
Hamiltonian is given by:

$$
H_{L S}=\frac{1}{2 m^{2} c^{2}} \frac{1}{r} \frac{d V(r)}{d r} \mathbf{s} \cdot \mathbf{I}
$$

The Bi $6 p$ wave function consists of a radial part $R$ and an angular part. For the angular part,

$$
\begin{gathered}
\langle\mathbf{s} \cdot \mathbf{l}\rangle=\frac{1}{2}\left(\left\langle\mathbf{j}^{2}\right\rangle-\left\langle\mathbf{l}^{2}\right\rangle-\left\langle\mathbf{s}^{2}\right\rangle\right)=\frac{\hbar^{2}}{2}[j(j+1)-l(l+1)-s(s+1)] \\
\Delta\langle\mathbf{s} \cdot \mathbf{l}\rangle=\langle\mathbf{s} \cdot \mathbf{l}\rangle_{j=3 / 2}-\langle\mathbf{s} \cdot \mathbf{l}\rangle_{j=1 / 2}=\frac{3 \hbar^{2}}{2} \\
\Delta \mathcal{E}_{6 p}=\Delta\left\langle H_{L S}\right\rangle=\frac{3 \hbar^{2}}{4 m^{2} c^{2}}\left\langle R\left|\frac{1}{r} \frac{d V(r)}{d r}\right| R\right\rangle=\frac{3 \hbar^{2} Z e^{2}}{4 m^{2} c^{2}}\left\langle R\left|\frac{1}{r^{3}}\right| R\right\rangle
\end{gathered}
$$

The ratio of the splitting for the alloy and atomic cases is given by

$$
\frac{\Delta \mathcal{E}\left(k_{x}\right)}{\Delta \mathcal{E}_{6 p}}=\frac{\frac{Z e^{2} \hbar^{2} k_{x} \Delta z}{2 m^{2} c^{2}}\left\langle u_{0}\left|\frac{1}{r^{3}}\right| u_{0}\right\rangle}{\frac{3 \hbar^{2} Z e^{2}}{4 m^{2} c^{2}}\left\langle R\left|\frac{1}{r^{3}}\right| R\right\rangle}=\frac{2 k_{x} \Delta z}{3} \frac{\left\langle u_{0}\left|\frac{1}{r^{3}}\right| u_{0}\right\rangle}{\left\langle R\left|\frac{1}{r^{3}}\right| R\right\rangle} \simeq \frac{2 k_{x} \Delta z}{3}
$$

In both cases, the matrix element integral is dominated by the region near the Bi nucleus, and the relevant wave function is $\mathrm{Bi} 6 p$, although for the solid case, there is some mixing with other orbitals, such as the $6 s$ and the Ag valence orbitals. The ratio of the matrix elements should be roughly unity. The results show that to first order, the band splitting is proportional to $\Delta z$ and $k_{x}$, and the splitting in the alloy can be as large as the atomic case. Putting in the value for the atomic splitting, 


$$
\Delta \mathcal{E}\left(k_{x}\right) \simeq 1.7 k_{x} \Delta z
$$

where energy is measured in $\mathrm{eV}$, and wave vector is measured in $\AA^{-1}$. The actual buckling is 0.65 $\AA^{-1}$. So

$$
\Delta \mathcal{E}\left(k_{x}\right) \simeq 1.1 k_{x}
$$

These approximate results are in good semi-quantitative agreement with the experiment and the DFT calculation (see Fig 2, where the experimental value for the coefficient in Eq. (19) is 3.05 and the first-principle value is 2.56 ). It should be noted that the approximation works only when the structural buckling $\Delta z$ is significantly smaller than the Bi atomic radius ( 1.6 $\AA)$. When $\Delta z$ becomes larger than the $\mathrm{Bi}$ atomic radius, the $\mathrm{Bi}$ atoms become effectively isolated from the Ag surface. Consequently, the band splitting deviates from the linear relation as shown in Fig. 2(b) and reduces gradually toward zero as $\Delta z$ increases further.

The above first-order analysis shows how the splitting in the alloy case can be related to the atomic case. This is possible because the spin-orbit interaction is dominated by the strong Coulomb field around the nucleus, which is essentially the same for a free atom and an atom in a solid. The main difference between the two cases is the mixing of other orbitals in the alloy case, but the effects are minor. For the alloy case, the splitting is zero at the zone center and again at the M-bar point because of symmetry requirements.

For first-principles band structure calculations, it is easier (and usual) to replace the core potential with a pseudopotential. The Hamiltonian transforms from 


$$
H=\frac{p^{2}}{2 m}+V(\mathbf{r})+\frac{1}{2 m^{2} c^{2}} \frac{1}{r} \frac{d V(r)}{d r} \mathbf{s} \cdot \mathbf{I}
$$

to

$$
H=\frac{p^{2}}{2 m}+V^{l o c}(r) \delta\left(\mathbf{r}-\mathbf{r}^{\prime}\right)+\sum_{l} V_{l}\left(\mathbf{r}, \mathbf{r}^{\prime}\right)+\Delta V_{l}^{S O}\left(\mathbf{r}, \mathbf{r}^{\prime}\right) \mathbf{s} \cdot \mathbf{I}
$$

where the second term is the local pseudopotential, the third term is the nonlocal pseudopotential, and the fourth term is the spin orbit interaction. It is important to recognize that the pseudopotential for the spin-orbit term has a very different form than the all-electron case.

$$
\Delta V_{l}^{S O}\left(\mathbf{r}, \mathbf{r}^{\prime}\right) \neq \frac{1}{2 m^{2} c^{2}} \frac{1}{r} \frac{d V(r)}{d r} \delta\left(\mathbf{r}-\mathbf{r}^{\prime}\right)
$$

If one simply replaces the all electron potential $V$ on the right-hand side of the above expression by the local and/or nonlocal pseudopotential, the results can be way off. The spin-orbit effect can be accounted for under the framework of pseudopotentials only when relativistic correction is treated properly by taking a different potential for each $j$ channel [23].

For a spin-split surface state such as the one observed on the noble metal surfaces $[2,13]$, one might be tempted to replace the all-electron potential $V$ in

$$
H_{L S}=\frac{\hbar}{4 m^{2} c^{2}} \boldsymbol{\sigma} \cdot \nabla V \times \mathbf{p}
$$

by the local pseudopotential as a first approximation,. One might further take $\nabla V$ to be the gradient of the surface potential step (work function), and the result would allow a straightforward estimate of the spin-orbit splitting. However, this simplification is unjustified and 
the estimated surface band splitting based on this naïve model is generally several orders of magnitude too small [14]. The large discrepancy has been noted before, and is not surprising. It comes about because the strong inner core potential is removed in the pseudopotential transformation. The resulting pseudopotential, not the real potential, depends on $j=l \pm 1 / 2$; the difference potential for the two $j$ values gives rise to the spin-orbit splitting [24].

\section{Circular dichroism in ARPES of the spin-polarized surface states in Bi/Ag}

Dichroic patterns from circularly-polarized ARPES measurements have been proven to be sensitive to the spin polarization of the surface states $[16,17,18,19,20]$. However due to the complication in the photoemission process it remains a challenge to extract the spin information from the measured dichroic patterns. The giant Rashba spin splitting of the surface states in $\mathrm{Bi} / \mathrm{A}$ is an ideal test ground for the basic physics of dichroism based on circularly polarized ARPES in terms of electromagnetic interactions with surface charge and spin. We show that the strong dichroic patterns in $\mathrm{Bi} / \mathrm{Ag}$ arise from the interference between two surface photoemission contributions, the Rashba interaction [17] and an often-neglected surface photoemission from $\nabla \cdot \mathbf{A}[25,26,27]$, as demonstrated by excellent agreement between a parameter-free calculation and experiment.

The experimental geometry is presented in Fig. 3(a) [15]. We take the normal of the

sample to be along $\hat{\mathbf{z}}$ and the $\bar{\Gamma} \bar{M}$ direction to be along $\hat{\mathbf{x}}$. The photon beam incidents in the $x z$ plane at an angle of $\theta \approx 60^{\circ}$. The polarization vector of vertically-polarized (VP) light is along $\hat{\mathbf{y}}$, and that of the horizontally polarized (HP) light lies within the $x z$ plane; here, "vertical" and "horizontal" refer to the plane of the electron storage ring used as the photon 
source. A schematic band diagram for the surface alloy (without the underlying quantum well states), assuming a large spin splitting appropriate for Bi/Ag, is presented in Fig. 3(b) with the red and blue colors indicate opposite Rashba spin directions $\pm \boldsymbol{k} \times \hat{\mathbf{z}}$. The spectrum taken along $\overline{\mathrm{M}}-\bar{\Gamma}-\overline{\mathrm{M}}$ form Bi/Ag prepared on a 20-ML $\mathrm{Ag}(111)$ film is shown in Fig. 3(c). A large Rashba splitting is clearly seen in the spectrum. The quantum well bulk subbands of the underlying $\mathrm{Ag}$ film are relatively weak but evident.

Photoemission is governed by the effective interaction Hamiltonian

$$
\Delta H \propto \mathbf{A} \cdot \nabla+\frac{1}{2} \nabla \cdot \mathbf{A}+\alpha \mathbf{s} \cdot \mathbf{A} \times \nabla V
$$

where $\mathbf{A}$ is the vector potential of the incident light. The first term corresponds to dipole transition and is the dominant contribution for bulk direct transitions governed by momentum conservation in all three dimensions. It is generally negligible for surface and quantum well states away from direct transitions as is the current case for the Ag bulk states. The second term, known as the surface-photoemission term, is important only at the surface where the dielectric function $\varepsilon$ and hence the vector potential $\mathbf{A}$ are discontinuous [27,28]. The discontinuity results in a $\nabla \cdot \mathbf{A}$ peaked around the surface. The third term depends on the spin $\mathbf{s}$ explicitly and stems from the Rashba interaction [17], where the canonical momentum depends on A. It also peaks around the surface because of the SIA at the surface as discussed in the previous section. 
(a)

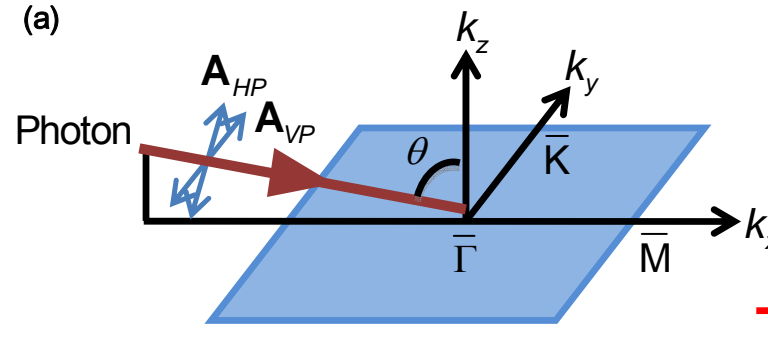

(b)

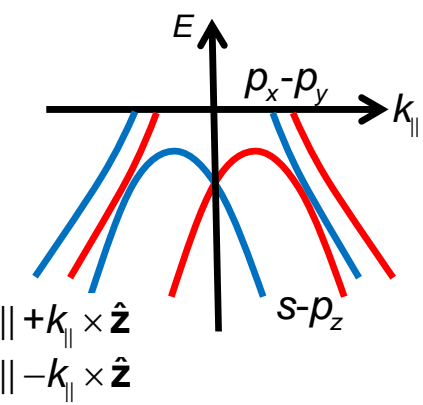

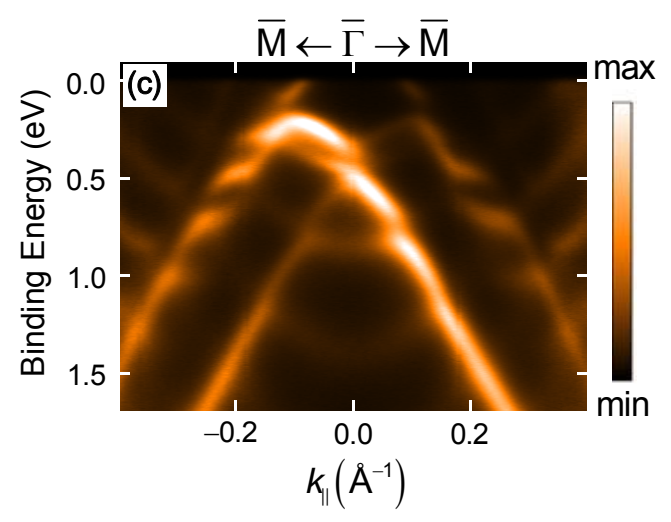

FIG. 3. Experimental geometry and an ARPES map from Bi/Ag(111) taken with HP light at $22 \mathrm{eV}$. (a) Experimental geometry. The normal of the sample surface is along $\hat{\mathbf{z}}$ and the vertical direction is along $\hat{\mathbf{y}}$ in the laboratory frame of reference. (b) Schematic band dispersion relations and spin textures. (c) Photoemission results taken along $\overline{\mathrm{M}}-\bar{\Gamma}-\overline{\mathrm{M}}$ for Bi/Ag prepared on a 20-layer $\mathrm{Ag}(111)$ film.

With the first term in Eq. (24) ignored, the photoemission matrix element becomes

$$
\left\langle\Psi_{f}|\Delta H| \Psi_{i}\right\rangle \propto\left\langle\Psi_{f}\left|\frac{1}{2} \nabla \cdot \mathbf{A}+\alpha \mathbf{s} \cdot \mathbf{A} \times \nabla V\right| \Psi_{i}\right\rangle
$$

In the classical limit, the product of the dielectric function and the z-component of the vector potential $\varepsilon A_{z}$ is continuous across the surface, while the in-plane components of the vector potential $A_{x, y}$ are continuous. Because $\varepsilon$ is discontinuous at the surface, $A_{z}$ must be discontinuous. As a result, $\nabla \cdot \mathbf{A}$ is proportional to $\delta(z)$ with the surface located at $z=0$. The matrix element involves an integration over all space. The result contains the product of the initial and final state 
wave functions at the surface, $\Psi_{f}^{*}(0) \Psi_{i}(0)$. Quantum mechanically, the delta function $\delta(z)$ is somewhat broadened with a width of about the screening length. For the second term in Eq. (25) arising from the Rashba interaction, the surface potential gradient is also a somewhat broadened delta function. After integration, it, too, contains the same $\Psi_{f}^{*}(0) \Psi_{i}(0)$ factor. Therefore the photoemission matrix element can be written as

$$
\left\langle\Psi_{f}|\Delta H| \Psi_{i}\right\rangle \propto\left[\frac{\hbar e}{2 m_{e}}\left(1-\frac{1}{\varepsilon}\right) A_{z}+\frac{\Delta z \beta e}{\hbar} \mathbf{A} \times \boldsymbol{\sigma} \cdot \hat{\mathbf{z}}\right] \Psi_{f}^{*}(0) \Psi_{i}(0) .
$$

The factor $\Psi_{f}^{*}(0) \Psi_{i}(0)$ can be taken to be part of the normalization factor and does not enter into the final calculations. The dichroic behavior is thus entirely determined by the relative strengths of the two terms. The photocurrent is given by

$$
I \propto|C|^{2} A_{z}^{2}+|D|^{2}\left|A_{y}\left\langle\sigma_{x}\right\rangle-A_{x}\left\langle\sigma_{y}\right\rangle\right|^{2}+\left(C^{*} D A_{z}^{*}\left(A_{y} \sigma_{x}-A_{x} \sigma_{y}\right)+\text { c.c. }\right)
$$

where $C=\hbar e(\varepsilon-1) / 2 m_{e} \varepsilon, D=e \beta \Delta z / \hbar$, and $A_{x, y, z}$ are the components of the vector potential. $\varepsilon=1.26+2.03 i$ for $\mathrm{Ag}$ at $22 \mathrm{eV}, \Delta z=0.65 \AA, \sigma$ is the expectation value of the spin polarization of the initial state, and the Rashba parameter $\beta$, related to the Rashba splitting, equals $3.05 \mathrm{eV}-\AA$ for $\mathrm{Bi} / \mathrm{Ag}$ from a fit to the band structure. It should be noted that the photon energy dependence as observed in the experiment can be attributed to variations of the dielectric function $\varepsilon$ provided that the photon energies chosen for the experiment are away from direct transitions. The spin polarization $\sigma$ depends on the wavevector as a consequence of the hybridization between the Rashba surface states and Ag bulk states. 


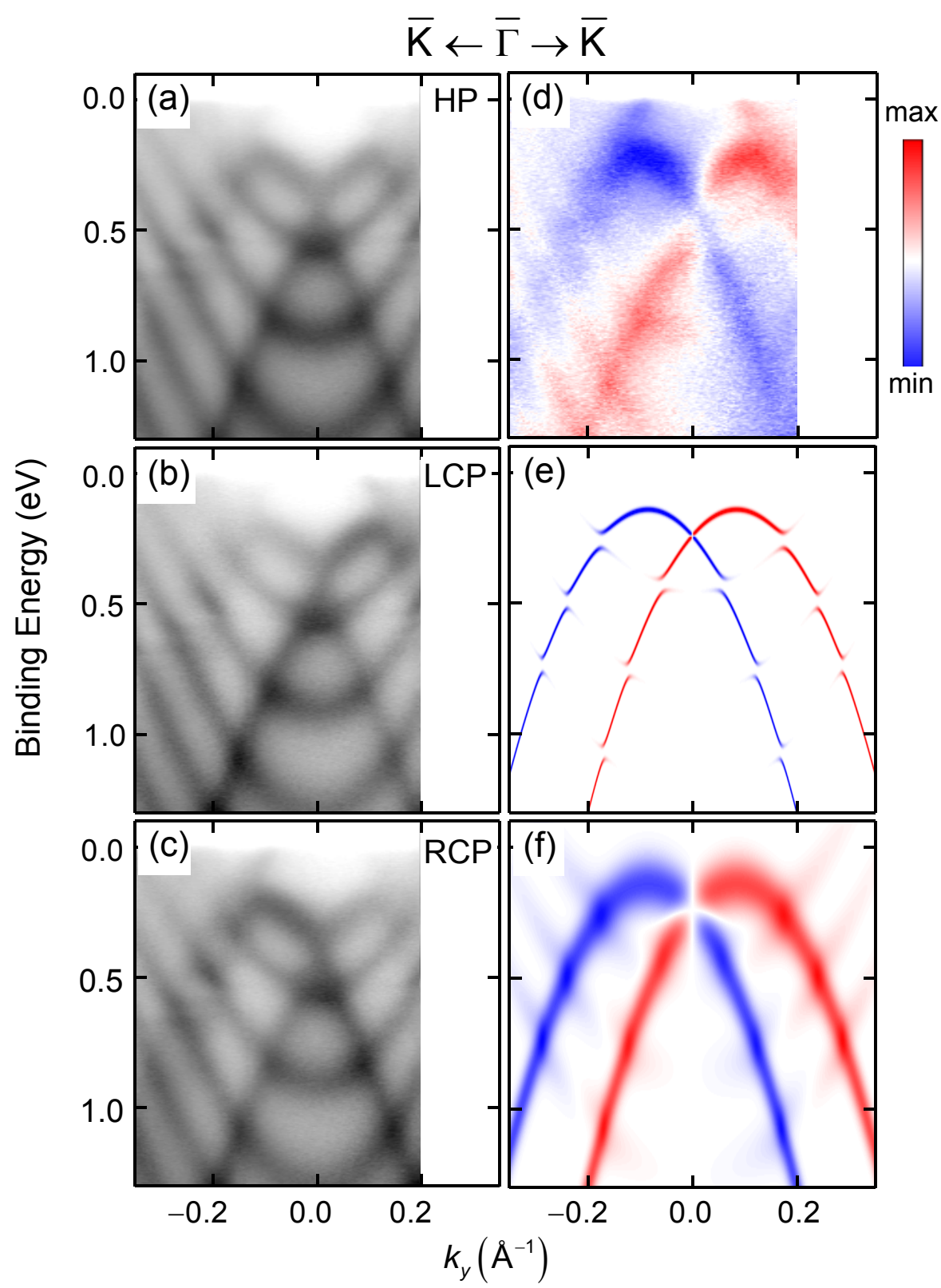

FIG. 4. Photoemission results taken from $\mathrm{Bi} / \mathrm{Ag}$ based on a $20 \mathrm{ML} \mathrm{Ag}$ film. The data were taken along $\overline{\mathrm{K}}-\bar{\Gamma}-\overline{\mathrm{K}}$ using $22 \mathrm{-eV}$ photons and (a) HP, (b) LCP, and (c) RCP polarization configurations. (d) Dichroic function extracted from the data. (e) Calculated band structure weighted by the spin polarization inherited from the Rashba split surface states using red and blue colors. (f) Calculated dichroic function.

Apart from an overall proportional constant, Eq. (27) contains no arbitrary or unknown 
parameters. To calculate the photoemission spectral map, we set up a Hamiltonian containing the spin-split bands (Eq. (1)) and a set of parabolic quantum well subbands positioned to correspond to the experiment. A spin-independent coupling term is included to generate the anticrossing gaps as seen in the experiment. Diagonalization of the Hamiltonian yields $\sigma$, from which the photoemission spectral maps are computed for various polarization configurations including HP, VP, left circular polarization (LCP), and right circular polarization (RCP) by taking the absolute square of the matrix element. Here, "left" and "right" refer to an observer viewing along the incident beam at the sample. A small constant lifetime broadening is included in the calculation.

Circular dichroism is apparent for measurements along $\overline{\mathrm{K}}-\bar{\Gamma}-\overline{\mathrm{K}}$, for which the system does not possess mirror symmetry about the $x z$ plane. While the HP map (Fig. 4(a)) shows equal intensities for the two spin-split bands, the LCP and RCP maps (Figs. 4(b-c)) show different intensities. In fact, the LCP and RCP maps appear to be left-right mirror images of each other about $\bar{\Gamma}$. To quantify this difference, we define a dichroic function

$$
\xi=\frac{I_{L}-I_{R}}{I_{L}+I_{R}}
$$

where $I_{L, R}$ are the photoemission intensities for LCP and RCP, respectively. The experimental results are presented in Fig. 4(d), where red and blue colors indicate positive and negative values of $\xi$, respectively. The maximum value of $\xi$ is about $\pm 30 \%$ at the top of each surface band and the sign of $\xi$ is opposite for the two bands. Fig 4(e) shows the theoretical spin polarization inherited from the Rashba split surface states. States with spin polarization along $+\hat{\mathbf{x}}$ and $-\hat{\mathbf{x}}$ directions are colored in red and blue, respectively. Near the hybridization gaps, the states 
transform smoothly from surface-like to bulk-like and the hybrid states are partially spin polarized. The calculated dichroic function (Fig. 4(f)) is in excellent agreement with the experimental pattern. The maximum value of $\xi$ is $\pm 38.9 \%$ from the calculation based on Eq. (27). It is somewhat higher than the experimental value; the difference can be attributed to the less than perfect polarization state of the LCP and RCP light and possibly to sample imperfections.

\section{Conclusions}

In this work we presented a theoretical analysis of the Rashba spin splitting of the surface states in $\mathrm{Bi} / \mathrm{Ag}$. The results demonstrated clearly the critical role played by the strong SOC associated with the Bi atoms and the SIA at the surface caused by surface buckling, and are in good accord with experimental measurements and DFT calculations. We also performed ARPES measurements using circularly polarized light on the same system. The good agreement between experiment and our calculation under various experimental conditions is a strong testimony of the theoretical treatment. The results confirm the strong spin texture of $\mathrm{Bi} / \mathrm{Ag}$. A key finding is that the surface-photoemission term derived from a nonzero $\nabla \cdot \mathbf{A}$ and the $\mathbf{A}$-dependent part of the Rashba interaction can interfere with each other and are both important for understanding the experimental results. The theoretical framework and experimental methodology reported here should be valuable for unraveling the electronic structure of complex systems including topological insulators, heavy-element compounds, superconductors, and strongly correlated systems in general $[9,10,29,30]$. 


\section{Acknowledgments}

This work is supported by the U.S. Department of Energy (Grant DE-FG02-07ER46383). The Synchrotron Radiation Center, where the photoemission data were taken, was funded by the University of Wisconsin-Madison. We acknowledge the U.S. National Science Foundation (Grant DMR-09-06444 and 13-05583) for partial support of beamline operations.

\section{References}

[1] Y. A. Bychkov and E. I. Rashba, J. Phys. C 17, 6039 (1984).

[2] S. LaShell, B. A. McDougall, and E. Jensen, Phys. Rev. Lett. 77, 3419 (1996).

[3] C. Ast et al., Phys. Rev. Lett. 98, 186807 (2007).

[4] H. Bentmann et al., Europhys. Lett. 87, 37003 (2009).

[5] G. Bihlmayer et al., Phys. Rev. B 75, 195414 (2007).

[6] L. Moreschini et al., Phys. Rev. B 80, 035438 (2009).

[7] I. Gierz et al., Phys. Rev. B 81, 245430 (2010).

[8] H. Bentmann et al., Phys. Rev. Lett. 108, 196801 (2012).

[9] M. Z. Hasan and C. L. Kane, Rev. Mod. Phys. 82, 3045 (2010).

[10] X.-L. Qi and S.-C. Zhang, Rev. Mod. Phys. 83, 1057 (2011).

[11] L. Petersen and P. Hedegård, Surf. Sci. 459, 49 (2000). 
[12] G. Bihlmayer et al., Surface Science 600, 3888 (2006).

[13] G. Nicolay, F. Reinert, S. Hüfner, and P. Blaha, Phys. Rev. B 65, 033407 (2001).

[14] B. Kim et al., Phys. Rev. B 85, 195402 (2012).

[15] G. Bian, X. Wang, T. Miller, and T.-C. Chiang, Phys. Rev. B 88, 085427 (2013).

[16] V. B. Zabolotnyy et al., Phys. Rev. B 76, 024502 (2007).

[17] Y. H. Wang et al., Phys. Rev. Lett. 107, 207602 (2011).

[18] Y. Liu et al., Phys. Rev. Lett. 107, 166803 (2011).

[19] Y. Ishida et al., Phys. Rev. Lett. 107, 077601 (2011).

[20] H. Mirhosseini and J. Henk, Phys. Rev. Lett. 109, 036803 (2012).

[21] G. Bian et al., Phys. Rev. Lett. 108, 186403 (2012)

[22] See http://www.nist.gov/pml/data/asd.cfm

[23] A. D. Corso and A. M. Conte, Phys. Rev. B 71, 115106 (2005).

[24] C. Hartwigsen, S. Goedecker, and J. Hutter, Phys. Rev. B 58, 3641 (1998).

[25] T. Miller, W. E. McMahon and T.-C. Chiang, Phys. Rev. Lett. 77, 1167 (1996).

[26] T. Miller, E. D. Hansen, W. E. McMahon and T.-C. Chiang, Surf. Sci. 376, 32 (1997).

[27] E. D. Hansen, T. Miller and T.-C. Chiang, Phys. Rev. Lett. 78, 2807 (1997).

[28] T.-C. Chiang, Surf. Sci. Rep. 39, 181 (2000).

[29] I. Gierz et al., Phys. Rev. Lett. 103, 046803 (2009).

[30] K. Yaji et al., Nat. Commun. 1:17 (2010). 


\section{Figures:}

FIG. 1. (a) Schematic structure of the Bi/Ag surface alloy used in the experiment. (b) Top view of the atomic structure. (c) Side view. Blue balls indicate Bi atoms and yellow balls stand for Ag atoms. (d) A photoemission spectrum from a Bi/Ag surface alloy along the $\bar{\Gamma}-\bar{M}$ direction taken with $22-\mathrm{eV}$ photons. The dashed curves are a guide to the eye for the upper Rashba split surface bands.

(a)

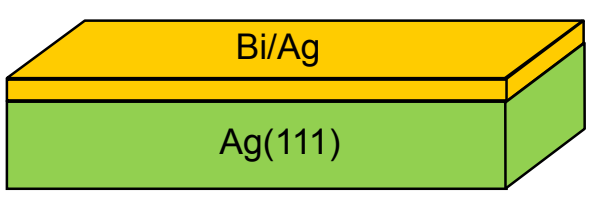

(b)

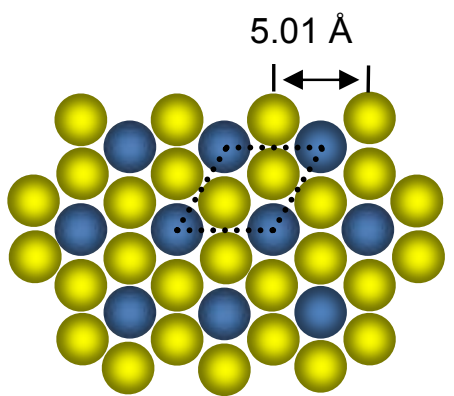

(c)

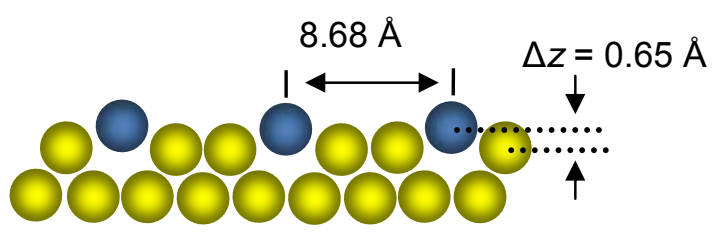

(d)

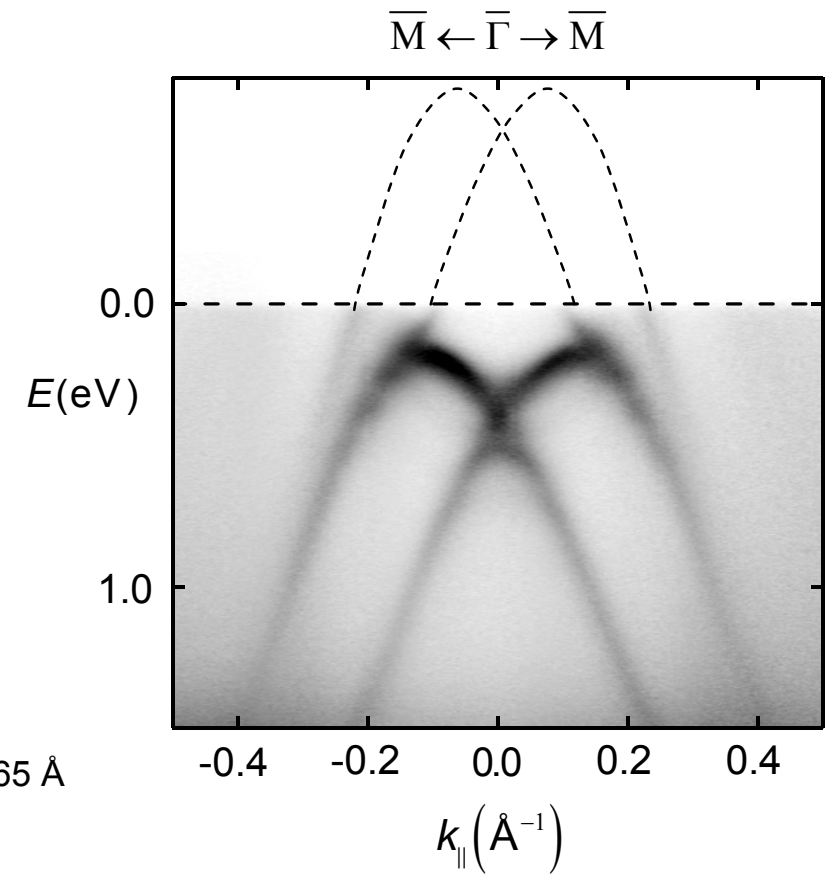


FIG. 2. (a) Calculated band structure of an isolated buckled layer of Bi/Ag. (b) Calculated Rashba momentum shift in the $p_{x, y}$ bands as a function of $\Delta z$.

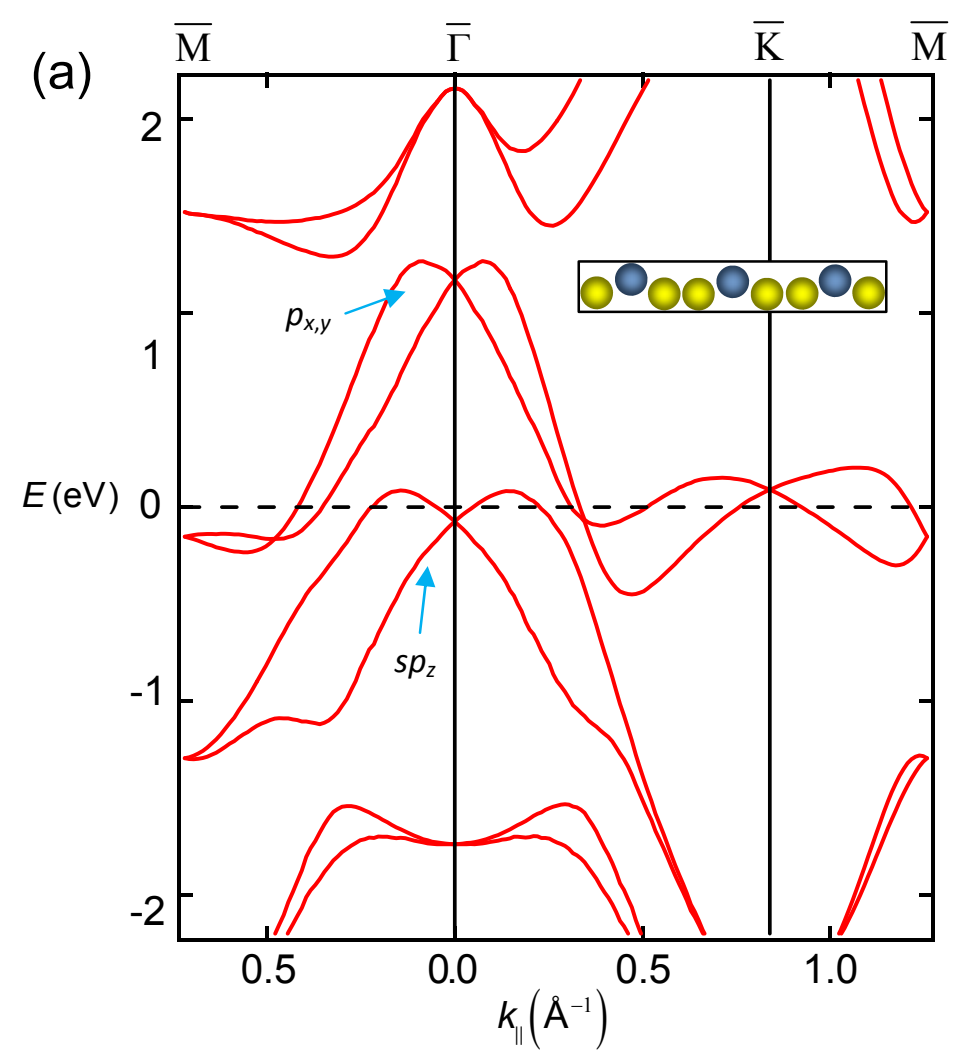

(b)

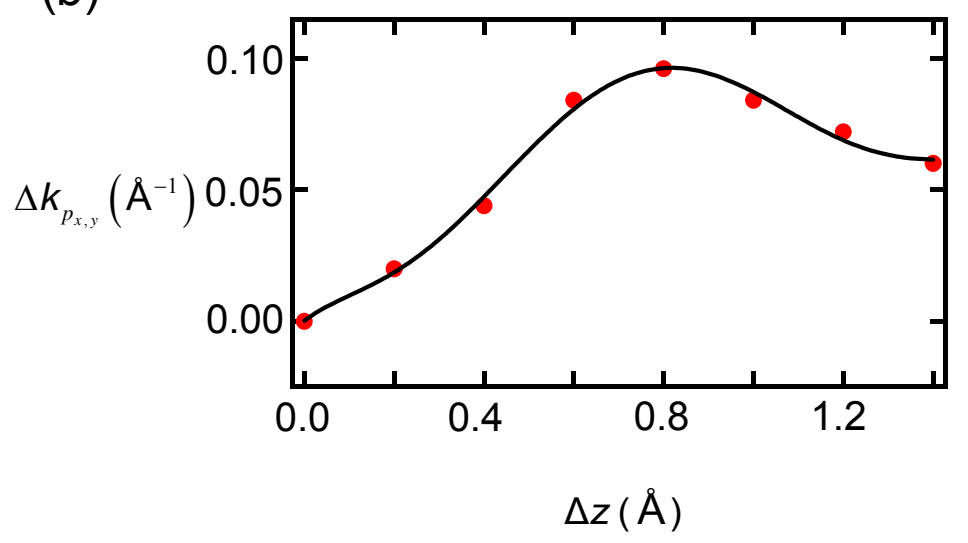


FIG. 3. Experimental geometry and an ARPES map from Bi/Ag(111) taken with HP light at $22 \mathrm{eV}$. (a) Experimental geometry. The normal of the sample surface is along $\hat{\mathbf{z}}$ and the vertical direction is along $\hat{\mathbf{y}}$ in the laboratory frame of reference. (b) Schematic band dispersion relations and spin textures. (c) Photoemission results taken along $\overline{\mathrm{M}}-\bar{\Gamma}-\overline{\mathrm{M}}$ for $\mathrm{Bi} / \mathrm{Ag}$ prepared on a 20-layer $\mathrm{Ag}(111)$ film.

(a)

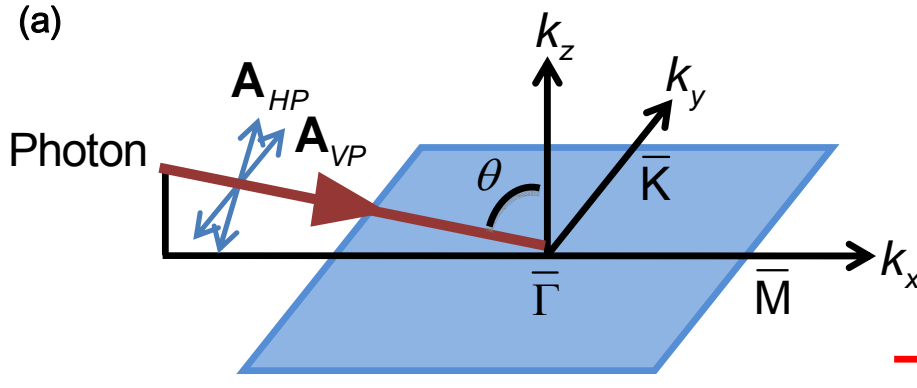

(b)

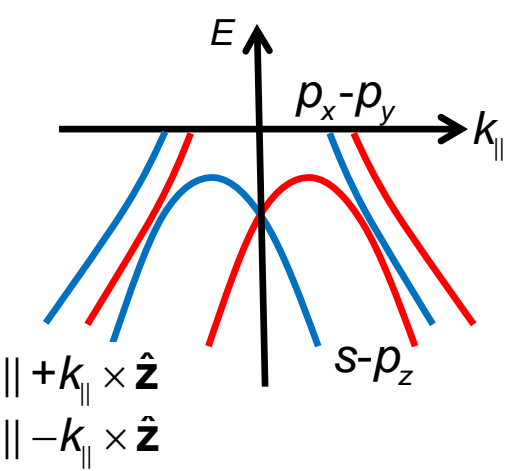

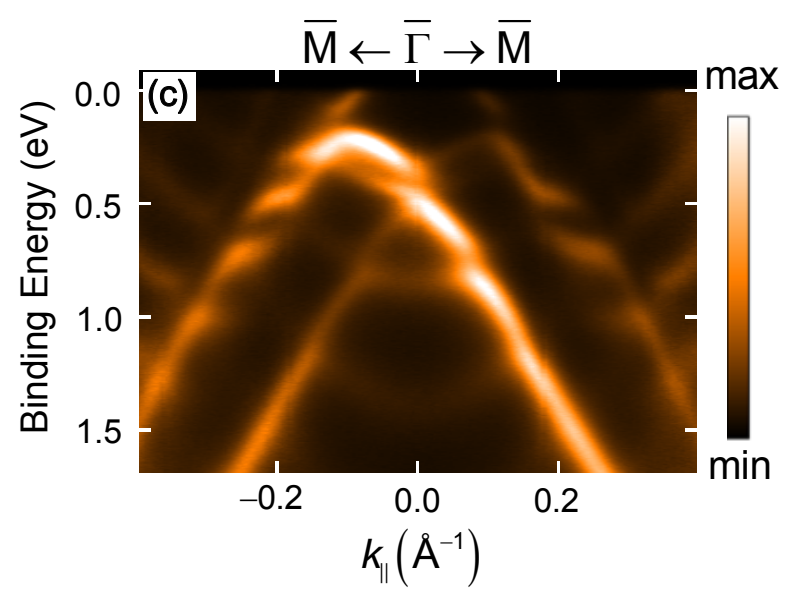


FIG. 4. Photoemission results taken from Bi/Ag based on a $20 \mathrm{ML} \mathrm{Ag} \mathrm{film.} \mathrm{The} \mathrm{data} \mathrm{were}$ taken along $\overline{\mathrm{K}}-\bar{\Gamma}-\overline{\mathrm{K}}$ using $22-\mathrm{eV}$ photons and (a) HP, (b) LCP, and (c) RCP polarization configurations. (d) Dichroic function extracted from the data. (e) Calculated band structure weighted by the spin polarization inherited from the Rashba split surface states using red and blue colors. (f) Calculated dichroic function.

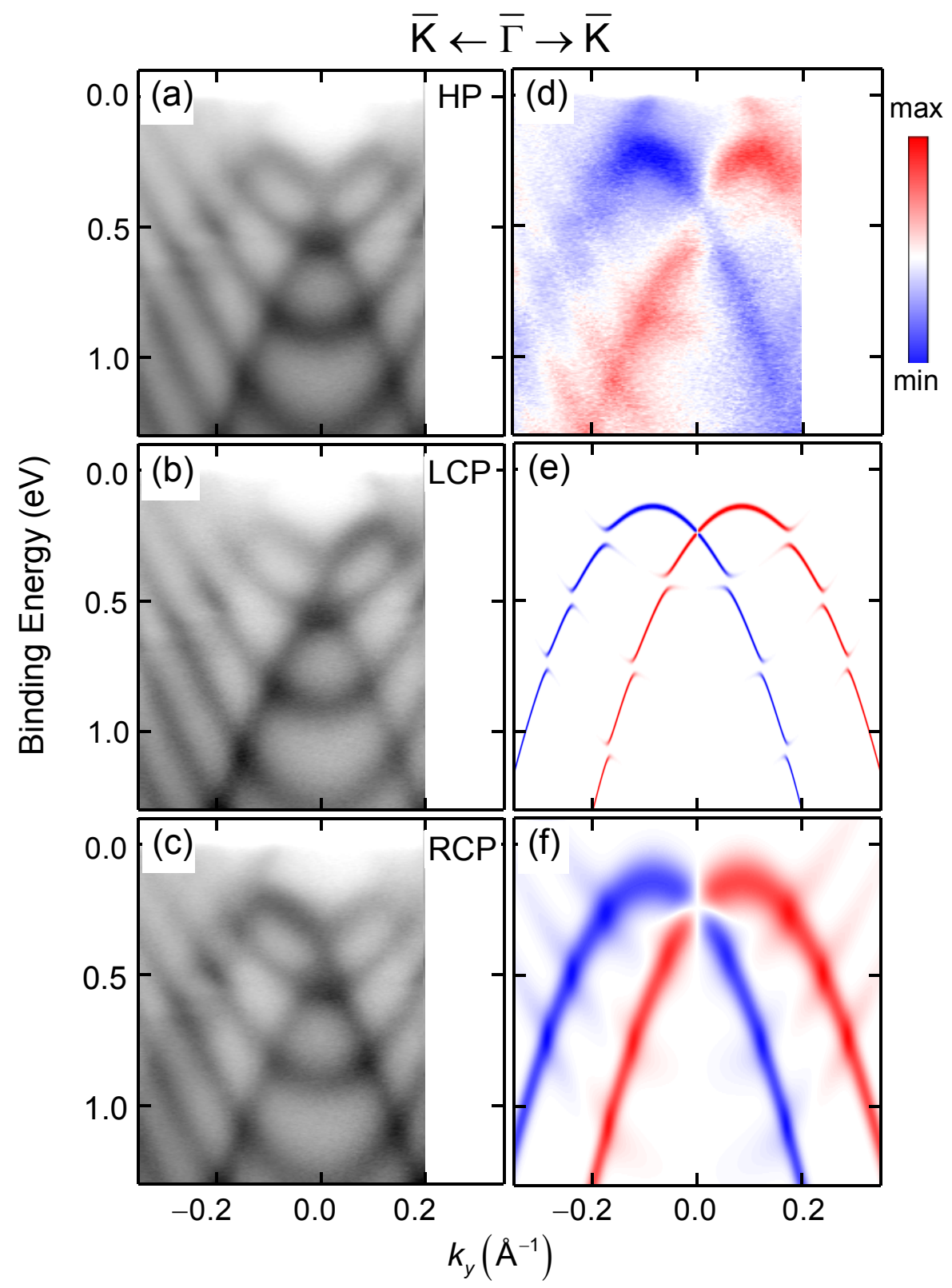


(a)

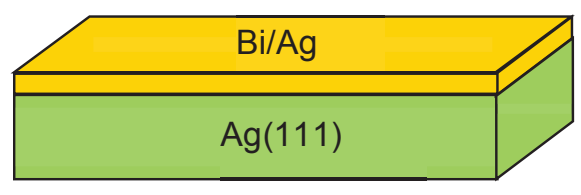

(b)

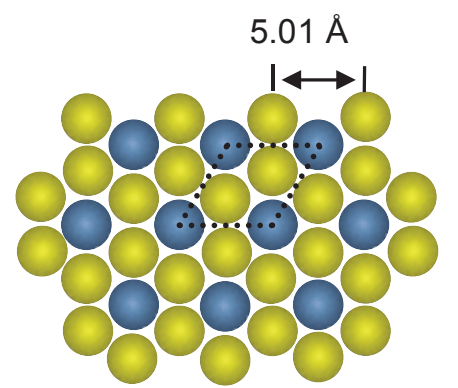

(c)

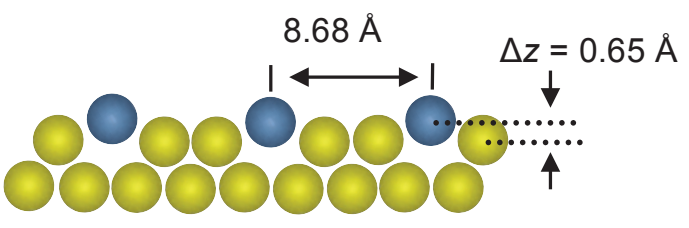

(d)

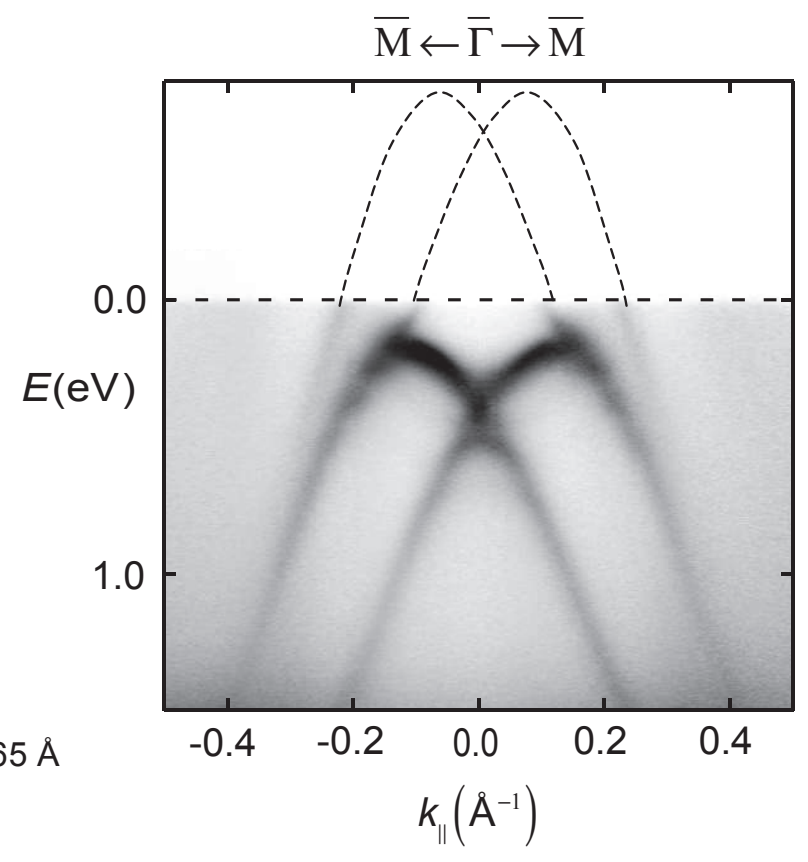




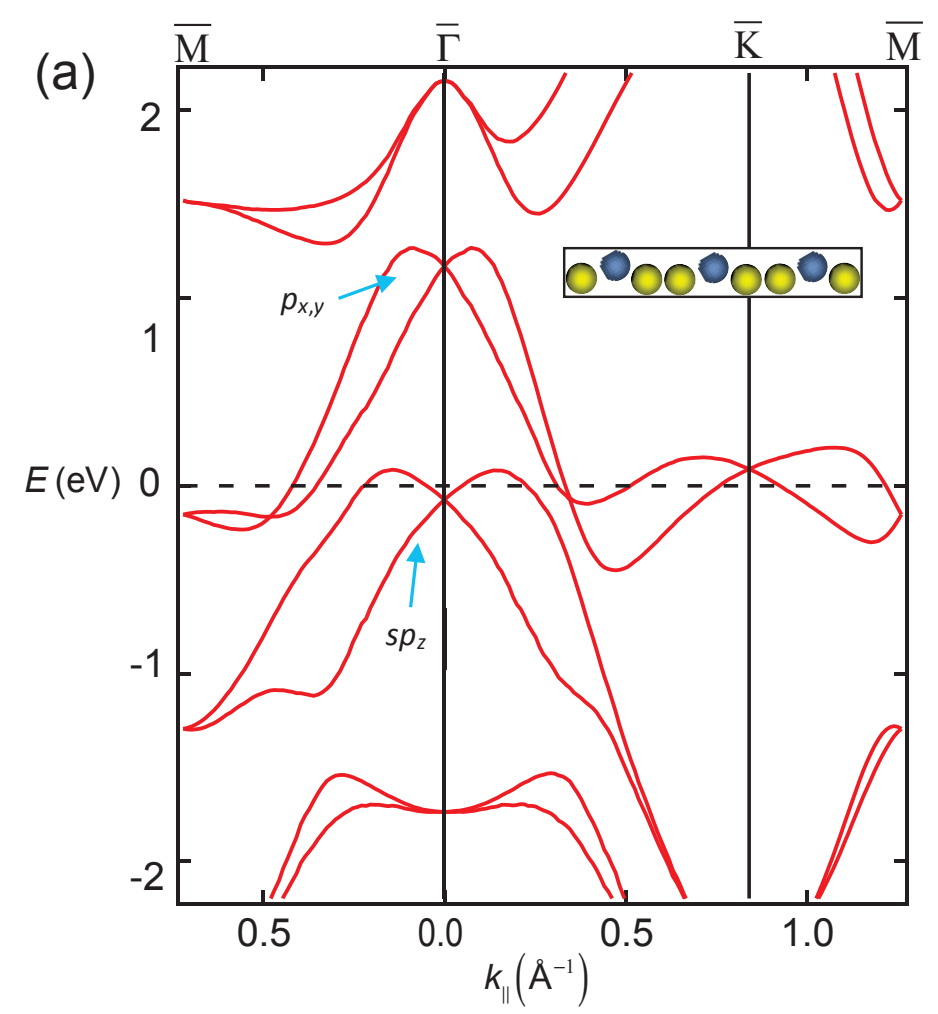

(b)

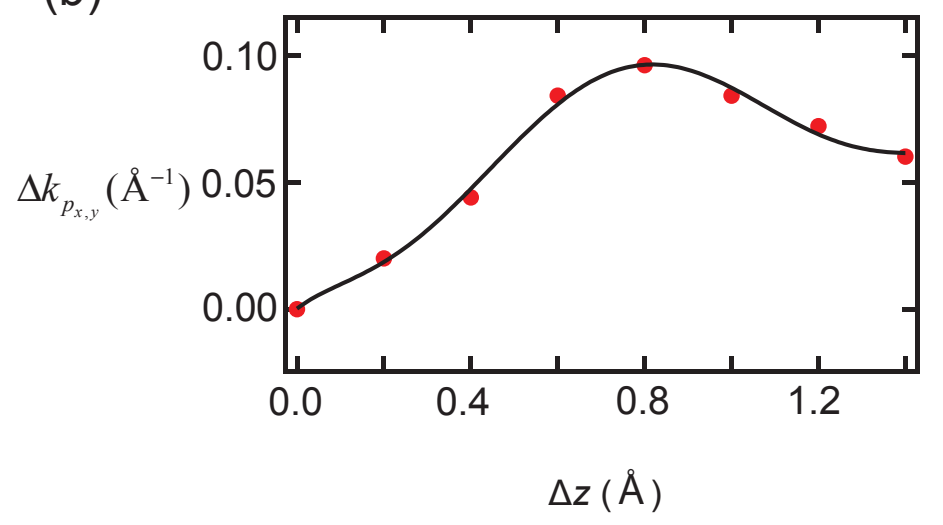


(b)$$
-\mathbf{s} \|-k_{\|} \times \hat{\mathbf{z}}
$$

(a)
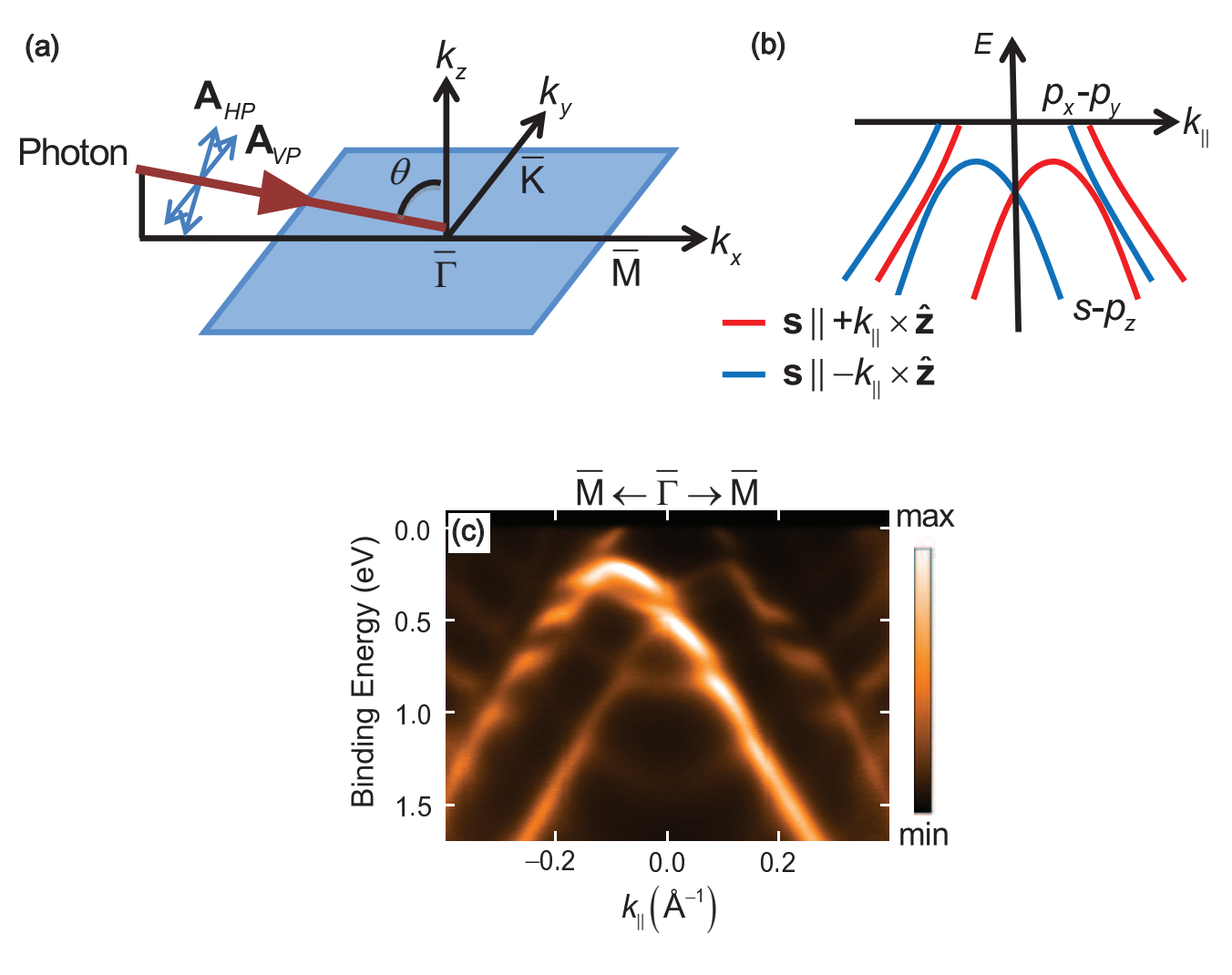

Figures 


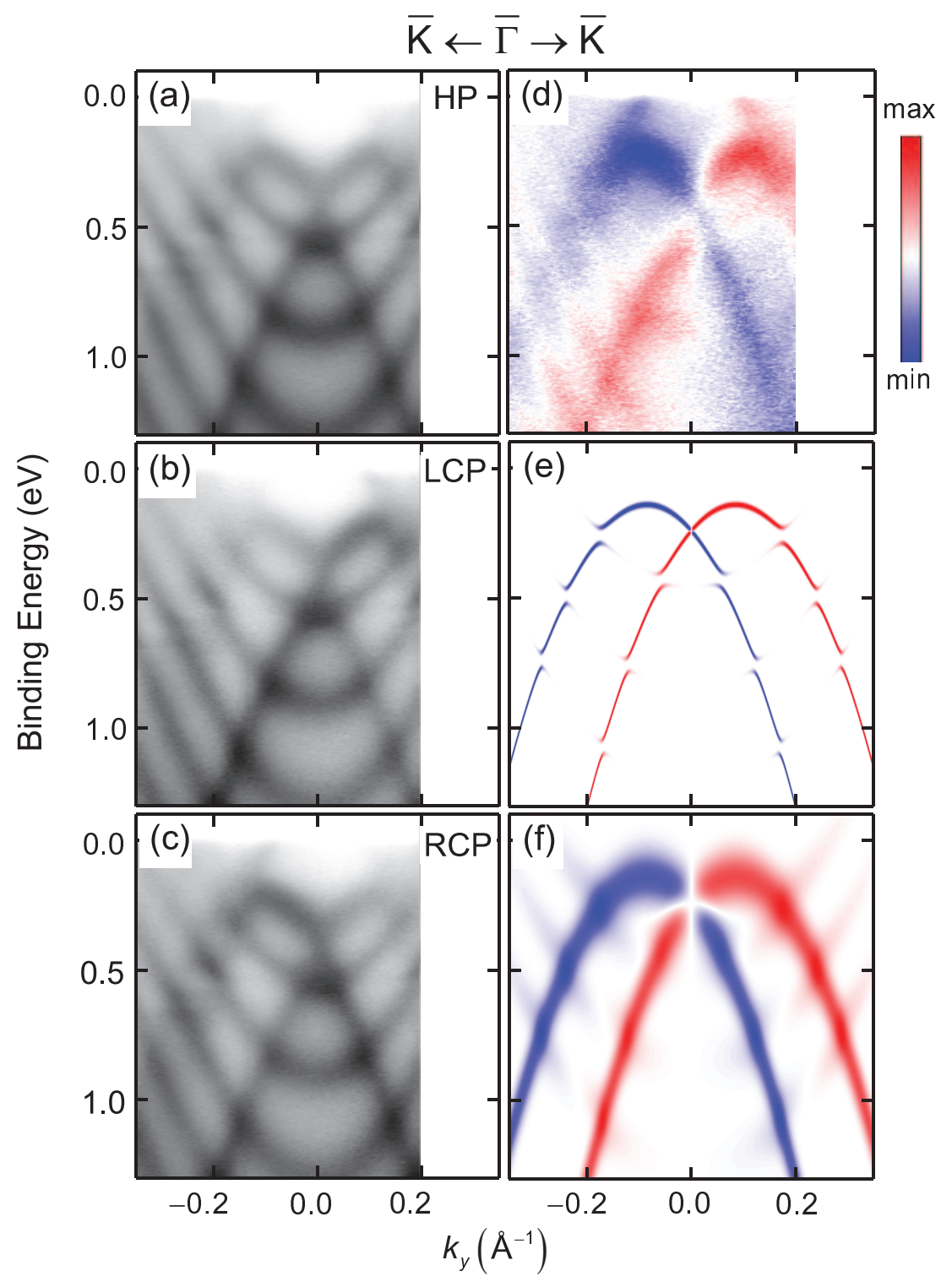

